\title{
REVISTA BRASILEIRA DE
}

AGRICULTURA IRRIGADA

Revista Brasileira de Agricultura Irrigada v.5, $\mathrm{n}^{\circ} .1, \mathrm{p} .48-53,2011$

ISSN 1982-7679 (On-line)

Fortaleza, CE, INOVAGRI - http://www.inovagri.org.br/rbai

Protocolo 03909 - 24/08/2010 Aprovado em 03/03/2011

\section{PROBABILIDADES QUINZENAIS DE OCORRÊNCIA DE TEMPERATURAS MÁXIMAS DO AR NA CIDADE DE IGUATU, BRASIL}

\author{
Eliakim Martins Araújo우 Efraim Martins Araújo루 Joaquim Branco de Oliveira; \\ Eder Ramon Feitosa Lêdo ${ }^{4}$; Paula Carneiro Viana ${ }^{5}$; Mairton Gomes Silva ${ }^{6}$
}

\footnotetext{
${ }^{1}$ Centro de Ciências Agrárias, Departamento de Engenharia Agrícola, UFC, Campus do Pici, 60356-000, Fortaleza - CE; eliakim.araujo@bol.com.br;

${ }^{2}$ Monitor de Topografia, Laboratório de Geoprocessamento, Aluno do Curso de Tecnologia em Irrigação e

Drenagem do IFCE - Campus Iguatu; efraimirrigacao@gmail.com;

${ }^{3}$ Doutorando em Fitotecnia, Prof. MSc. do IFCE - Campus Iguatu . Email:

joaquimbrancodeoliveira@gmail.com;

${ }^{4}$ Bolsista, Aluno do Curso de Tecnologia em Irrigação e Drenagem do IFCE - Campus Iguatu; eder_ramon@hotmail.com;

${ }^{5}$ Aluna do Curso de Tecnologia em Irrigação e Drenagem do IFCE - Campus Iguatu; paulinhatmgm@hotmail.com;

${ }^{6}$ Bolsista FUNCAP, Aluno do Curso de Tecnologia em Irrigação e Drenagem do IFCE - Campus Iguatu; mairtong@hotmail.com;
}

\section{RESUMO}

Este estudo teve como objetivo calcular as probabilidades de ocorrência das temperaturas máximas do ar da cidade de Iguatu, Ceará, Brasil, em escala quinzenal para todos os meses do ano a diferentes níveis de probabilidade. Foram utilizados dados diários de temperatura máxima do ar, obtidos junto ao $3^{\circ}$ Distrito do Instituto Nacional de Meteorologia (INMET). A distribuição utilizada foi a Log-Normal e seu desempenho foi testado segundo os testes de aderência Qui-quadrado e Kolmogorov-Smirnov. Os níveis de probabilidade utilizados foram 25, 50, 75 e 90\%, representando um período de retorno de 1,3; 2; 4 e 10 anos, respectivamente. A distribuição Log-Normal obteve ajuste considerado satisfatório e pode ser utilizada para a representação dos dados utilizados no estudo segundo ambos os testes de aderência. Após a aplicação da distribuição Log-Normal foi possível gerar tabelas com os valores esperados de temperatura máxima do ar para os diferentes níveis de probabilidade. Os resultados das tabelas evidenciam que para todo o ano, em escala quinzenal, a temperatura máxima de Iguatu para todos os níveis de probabilidade estudados, será maior que $30{ }^{\circ} \mathrm{C}$.

PALAVRAS-CHAVE: Log-Normal, testes de aderência, níveis de probabilidade.

\section{OCCURRENCE PROBABILITY OF MAXIMUM AIR TEMPERATURE IN SCALE OF FIFTEEN DAYS IN IGUATU CITY, BRAZIL}


PROBABILIDADES QUINZENAIS DE OCORRÊNCIA DE TEMPERATURAS MÁXIMAS DO AR NA CIDADE DE IGUATU, BRASIL

\begin{abstract}
This study aimed to calculate the probabilities of occurrence of maximum air temperatures in Iguatu city, Ceará, Brazil, on a scale of fifteen days for each month of the year at different levels of probability. Were used daily data of maximum air temperature, obtained from the 3rd District of the Instituto Nacional de Meteorologia (INMET). The distribution used was the Log-Normal and its performance was tested according to the Chi-square and KolmogorovSmirnov test. The probability levels used were $25,50,75$ and $90 \%$, representing a payback period of 1,3, 2, 4 and 10 years respectively. The Log-Normal distribution fit obtained satisfactory and can be used for the representation of the data used in the study according to both tests of adhesion. After applying the Log-normal distribution was possible to generate tables with the values of maximum air temperature expected for different levels of probability. The results of the tables show that for every month of the year, on a scale of fifteen days, to a maximum temperature of Iguatu at all levels of probability studied, will be greater than $30{ }^{\circ} \mathrm{C}$.
\end{abstract}

KEYWORDS: Log-Normal, adherence tests, probability levels.

\section{INTRODUÇÃO}

A temperatura é um dos mais importantes elementos meteorológicos, pois a mesma traduz os estados energéticos e dinâmicos da atmosfera e consequentemente revela a circulação atmosférica, sendo capaz de facilitar e/ou bloquear os fenômenos atmosféricos. Portanto, é de fundamental importância as medições da temperatura do ar, em qualquer instante, principalmente naqueles horários recomendados pela Organização Meteorológica Mundial, incluindo neste caso as temperaturas do ar máxima e mínima, ocorridas em um período de 24 horas, e na impossibilidade destas ultimas medidas, deve-se recorrer a métodos de estimativas (DANTAS et al., 2000).

O estudo das distribuições de variáveis, ao longo do tempo, como um meio de compreender os fenômenos meteorológicos, para determinar seus padrões de ocorrência e permitir uma previsibilidade razoável do comportamento climático de uma região, é uma ferramenta de grande valor para o planejamento $\mathrm{e}$ gestão de inúmeras atividades agropecuárias e humanas (ASSIS et al., 2004).

$\mathrm{O}$ uso de funções densidade de probabilidade está diretamente ligado à natureza dos dados a que estas funções estão sendo relacionadas. Algumas têm boa capacidade de estimação para pequeno número de dados, outras requerem grande série de observações. Devido ao número de parâmetros de sua equação, algumas podem assumir diferentes formas, enquadrando-se em um número maior de situações, ou seja, são mais flexíveis. Desde que respeitado o aspecto da representatividade dos dados, as estimativas dos seus parâmetros para uma determinada região, podem ser estabelecidas como de uso geral, sem prejuízo da precisão na estimação da probabilidade (CATALUNHA et al., 2002).

A distribuição de probabilidade LogNormal é muito utilizada em hidrologia e climatologia (MELLO et al., 1994). Essa distribuição considera que os logaritmos das variáveis aleatórias seguem uma distribuição Normal (FRIZZONE, 1979 citado por LYRA et al., 2006).

Araújo et al. (2010) avaliaram a distribuição de probabilidade que melhor se ajusta a series diárias de temperatura máxima da cidade de Iguatu no Ceará, utilizando seis diferente distribuições de probabilidade, constatando que a distribuição Log-Normal obteve desempenho considerado bom.

Em Lavras - MG, a distribuição LogNormal mostrou-se adequada para 
PROBABILIDADES QUINZENAIS DE OCORRÊNCIA DE TEMPERATURAS MÁXIMAS DO AR NA CIDADE DE IGUATU, BRASIL

predição das precipitações prováveis apenas nos meses de maior precipitação (SAMPAIO et al., 1999).

O objetivo desse estudo foi determinar as temperaturas do ar máximas prováveis para os períodos de retorno de $1,3,2,4$ e 10 anos, para a escala de quinze dias na cidade de Iguatu (CE), utilizando-se a distribuição Log-Normal, analisando seu desempenho em relação aos dados.

\section{MATERIAL E METÓDOS}

Os dados de temperatura máxima do ar, utilizados para o ajuste da função distribuição de probabilidade, foram obtidos junto ao $3^{\circ}$ Distrito do Instituto Nacional de Meteorologia (INMET) de Iguatu (latitude: $6^{\circ} 22^{\prime} \mathrm{S}$, longitude $39^{\circ} 18^{\prime}$ W e altitude: $217,67 \mathrm{~m}$ ) do período de 1961 a 2005, totalizando uma série de 40 anos. A cidade de Iguatu acha-se situada no Semiárido do Nordeste brasileiro, compreendendo, segundo a classificação de Köppen, o tipo de clima: BSw'h', representando um clima muito quente, Semiárido, com estação chuvosa atrasada (SUDENE, 1973).

A homogeneidade dos dados foi testada segundo a metodologia proposta por Thom (1966), sendo constatado que a serie é homogênea. Em toda a série não foram encontradas falhas, fato esse que não exigiu nenhum tipo de tratamento especial aos dados. Os dados foram separados em escala quinzenal (período de 15 dias), agrupados em classes e realizaram-se os procedimentos de distribuição de frequência. Após os procedimentos de distribuição de freqüência utilizou-se a distribuição de probabilidade Log-Normal. Conforme Ribeiro et al. (2007), a função densidade da distribuição Log-Normal a dois parâmetros e a três parâmetros são representadas pela seguinte equação:

$$
\mathrm{F}(\mathrm{x})=\frac{1}{(\mathrm{x}-\mathrm{a}) \sigma \sqrt{2 \pi}} \mathrm{e}\left(-\frac{[\ln (\mathrm{x}-\mathrm{a})-\mu]^{2}}{2 \sigma^{2}}\right)
$$

Em que:

$\mathrm{F}(\mathrm{x})$ - função densidade de probabilidade da variável,

e - base do logaritmo neperiano,

$\mathrm{x}$ - valor da variável aleatória,

$\mu$ - média dos logaritmos da variável $x$,

$\sigma$ - desvio-padrão dos logaritmos da variável x,

a - limite inferior da amostra.

Para encontrar a probabilidade de que uma variável aleatória $\mathrm{x}$ tendo distribuição LogNormal, assuma valores entre $a$ e b $(\mathrm{a} \leq \mathrm{x} \leq \mathrm{b})$, tem-se:

$$
F(a \leq x \leq b)=\int_{a}^{b} \frac{1}{x \sigma \sqrt{2 \pi}} \exp \left(-\frac{[\ln (x-a)-\mu]^{2}}{2 \sigma^{2}}\right) d x
$$

O valor de "a" pode ser zero, quando se considera a distribuição Log-Normal a dois parâmetros, ou um valor mínimo da série, quando se considera Log-Normal a três parâmetros. O valor de "b" pode ser o da variável aleatória, quando se considera a probabilidade cumulativa de ocorrência daquele valor (HASTINGS; PEACOCK, 1975 citados por CATALUNHA et al., 2002).

Ao ajustar uma distribuição de probabilidade a um conjunto de dados, assume-se a hipótese de que a distribuição pode representar adequadamente o conjunto de informações. Para verificar o ajuste entre as Frequências de temperaturas máximas decendiais observadas e estimadas adotou-se os testes de Quiquadrado $\left(\chi^{2}\right)$ e Kolmogorov-Smirnov (KS), considerando os níveis de significância como sendo 5 e $20 \%$, respectivamente.

Ao final, foram geradas tabelas com os valores estimados de temperatura máxima do ar para níveis de 25, 50, 75 e 90\% de probabilidade de ocorrência e seu respectivo tempo de retorno para a escala decendial. 
PROBABILIDADES QUINZENAIS DE OCORRÊNCIA DE TEMPERATURAS MÁXIMAS DO AR NA CIDADE DE IGUATU, BRASIL

\section{RESULTADOS E DISCUSSÃO}

Através da análise de distribuição de frequência foi possível utilizar a função distribuição de probabilidade Log-Normal para a estimativa de ajuste dos dados de temperatura máxima para a escala quinzenal, da cidade de Iguatu. Entende-se por ajuste, a aproximação entre as frequências observadas e esperadas por cada função, ajustes esses que são dados pelos testes de aderência.

$\mathrm{Na}$ verificação do ajuste da distribuição Log-Normal a partir do teste Qui-quadrado (Tabela 1) observou-se que ela obteve um desempenho bom, podendo ser utilizada para representar os dados para dez meses do ano. Como no estudo de Araújo et al. (2010), a aderência das distribuição LogNormal para a analise dos dados segundo o teste $\chi^{2}$.

Tabela 1. Ajuste da distribuição de probabilidade Log-Normal aos dados de temperatura do ar máxima na cidade de Iguatu, na escala decendial, para o teste Qui-quadrado ao nível de 5\% de significância.

\begin{tabular}{lccccccccccccc}
\hline \multirow{2}{*}{$\begin{array}{l}\text { Distribuição de } \\
\text { probabilidade }\end{array}$} & Jan & Fev & Mar & Abr & Mai & Jun & Jul & Ago & Set & Out & Nov & Dez \\
\hline Log-Normal & $*$ & n.s. & n.s. & n.s. & n.s. & n.s. & n.s. & n.s. & n.s. & n.s. & n.s. & $*$ \\
\hline
\end{tabular}

Onde: n.s. - não significativo; *. - significativo.

Observando-se o ajuste da distribuição Log-Normal pelo teste KolmogorovSmirnov (Tabela 2), é possível notar que ela obteve um desempenho semelhante ao constatado pelo teste $\chi^{2}$, diferindo apenas nos meses em que ela não obteve ajuste. Em ambos os casos, o desempenho da distribuição é capaz de justificar a sua utilização para a estimativa da probabilidade de ocorrência, ou seja, os valores estimados de temperatura do ar em diferentes níveis de segurança para a cidade de Iguatu.

Tabela 2. Ajuste da distribuição de probabilidade Gumbel aos dados de temperatura do ar máxima na cidade de Iguatu, na escala decendial, para o teste Kolmogorov-Smirnov ao nível de $20 \%$ de significância.

\begin{tabular}{llllllllllllll}
\hline \multirow{2}{*}{$\begin{array}{l}\text { Distribuição de } \\
\text { probabilidade }\end{array}$} & Jan & Fev & Mar & Abr & Mai & Jun & Jul & Ago & Set & Out & Nov & Dez \\
\hline Log-Normal & n.s. & n.s. & n.s. & n.s. & n.s. & n.s. & n.s. & n.s. & n.s. & $*$ & n.s. & $*$ \\
\hline
\end{tabular}

Onde: n.s. - não significativo; *. - significativo.

Foi constatado, então, que a distribuição Log-Normal pode ser utilizada na estimativa da temperatura do ar máxima provável para Iguatu na escala quinzenal para os níveis de $25,50,75$ e $90 \%$ de probabilidade de ocorrência (Tabela 3), onde tais níveis de probabilidade representam os limites de ocorrência de valores iguais ou inferiores aos calculados, ou seja, de acordo com a Tabela 3, existem $90 \%$ de probabilidade de que o valor da temperatura do ar máxima média para o mês de janeiro em escala quinzenal não ultrapasse $35,1^{\circ} \mathrm{C}$. Tal valor também pode ser interpretado como se a cada dez anos, 
PROBABILIDADES QUINZENAIS DE OCORRÊNCIA DE TEMPERATURAS MÁXIMAS DO AR NA CIDADE DE IGUATU, BRASIL

nove apresentarão valor igual ou menor

que $35,1^{\circ} \mathrm{C}$.

Tabela 3. Diferentes níveis de probabilidade de ocorrência da temperatura do ar máxima em escala quinzenal para a cidade de Iguatu, segundo a distribuição Log-Normal.

\begin{tabular}{lcccc}
\hline Escala decendial para todos & \multicolumn{4}{c}{ Níveis de probabilidade \% } \\
\cline { 2 - 5 } os meses do ano & 25 & 50 & 75 & 90 \\
\hline Janeiro & 31,3 & 32,6 & 33,9 & 35,1 \\
Fevereiro & 30,7 & 31,6 & 32,6 & 33,5 \\
Março & 30,2 & 31,0 & 31,8 & 32,5 \\
Abril & 30,2 & 30,8 & 31,5 & 32,1 \\
Maio & 29,5 & 30,4 & 31,3 & 32,2 \\
Junho & 29,6 & 30,5 & 31,5 & 32,3 \\
Julho & 30,8 & 31,5 & 32,4 & 33,1 \\
Agosto & 32,5 & 33,2 & 33,8 & 34,4 \\
Setembro & 34,0 & 34,5 & 35,0 & 35,4 \\
Outubro & 34,8 & 35,3 & 35,8 & 36,3 \\
Novembro & 34,0 & 34,1 & 33,7 & 31,6 \\
Dezembro & 33,9 & 34,6 & 35,4 & 36,1 \\
\hline \multicolumn{1}{c}{ Período de retorno (anos) } & 1,3 & 2 & 4 & 10 \\
\hline
\end{tabular}

\section{CONCLUSÕES}

A distribuição de probabilidade LogNormal teve bom desempenho na estimativa de ocorrência da temperatura máxima do ar na cidade de Iguatu, segundo os testes de aderência Qui-quadrado e Kolmogorov-Sminorv. A construção de tabelas de probabilidade se mostrou uma ferramenta de grande valia no estudo do comportamento térmico de uma região.

\section{REFERÊNCIAS BIBLIOGRÁFICAS}

ARAÚJO, E. M.; SILVA, I. N.; OLIVEIRA, J. B., CAVALCANTE JÚNIOR, E. G.; ALMEIDA, B. M. Aplicação de seis distribuições de probabilidade a séries de temperatura máxima em Iguatu - CE. Revista Ciência Agronômica, Fortaleza, v. 41, n. 1, p. 3645, 2010.
ASSIS, J. P.; DOURADO NETO, D.; MANFRON, P. A.; MARTIN, T. N.; SPAROVEK, G.; TINM, L. C. Ajuste de séries históricas de temperatura e radiação solar global diária às funções densidade de probabilidade normal e log-normal, em Piracicaba, SP. Revista Brasileira de Agrometeorologia, Santa Maria, v. 12, n. 1, p. 113-121, 2004.

CATALUNHA, M. J.; SEDIYAMA G. C.; LEAL, B. G.; SOARES, C. P. B.; RIBEIRO, A. Avaliação de cinco funções densidade de probabilidade a séries de precipitação pluvial do Estado de Minas Gerais. Revista Brasileira de Agrometeorologia. Santa Maria, v. 10, n. 1, p. 153-162, 2002.

DANTAS, R. T.; NÓBREGA, R. S.; CORREIA, A. M; RAO, T. V. R. Estimativas das temperaturas máxima e mínima do ar em Campina Grande - PB. In: CONGRESSO BRASILEIRO DE 
METEOROLOGIA; RIO DE JANEIRO, 11. Rio de Janeiro. Anais... Rio de Janeiro. SBMET, 2000. p. 534-537.

LYRA, G. B.; GARCIA, B. I. L.; PIEDADE, S. M. S.; SEDIYAMA, G. C.; SENTELHAS, P. S. Regiões homogêneas e funções de distribuição de probabilidade da precipitação pluvial no Estado de Táchira, Venezuela. Pesquisa Agropecuária Brasileira, Brasília, v. 41, n. 2, p. 205-215, 2006.

MELLO, M. H. A.; ARRUDA, H. V.; ORTOLANI, A. A. Probabilidade de ocorrência de totais pluviais máximos horários em Campinas - São Paulo. Revista do Instituto Geológico, São Paulo, v. 15, n. 2, p. 59-67, 1994.

RIBEIRO, B. T.; AVANZI, J. C.; MELLO, C. R.; LIMA, J. M.; SILVA, M. L. N.
Comparação de distribuições de probabilidade e estimativa da precipitação provável para região de Barbacena, MG. Ciência e Agrotecnologia, Lavras, v. 31, n. 5, p. 1297-1302, 2007.

SAMPAIO, S. C.; CORRÊA, M. M.; SOUZA, M. R.; GUIMARÃES, J. C.; SILVA, A. M. Precipitação provável para o município de Lavras-MG, utilizando a distribuição Log-normal. Ciências e Agrotecnologia, Lavras, v. 23, n. 2, p. 382-389, 1999.

SUDENE. Levantamento Exploratório Reconhecimento de Solos do Estado do Ceará - Volume I. Recife: SUDENE, 1973. $301 \mathrm{p}$.

THOM, H.C.S. Some methods of climatological analysis. Geneva, World Meteorological Organization, 1966. 53p. 